\title{
EXPERIENCIA DE LAS ADOLESCENTES SOBRE EL APOYO FAMILIAR DURANTE EL EMBARAZO.
}

Mtra. Leticia Costro Durän* Mtra. Rosa Mario Ostiguín Meléndez**

"Exalumna de la primera generación de Moestria en Enfermeria de la UNAM, Profesora del Centro de Estudios Tecnológicos e Industriales No. 76 de la SEP

* Profesora del Carreratitular "A" de la ENEO UNAM

\section{Resumen}

América Latina observa una tasa de fecundidad anual aproximada de 100 partos por mil mujeres, entre 15 y 19 años. En México cada año se dan 450, 000 embarazos de madres adolescentes lo que representa el 25\% del total de gestaciones que se registran en todo el país, con un alto impacto social, biológico y psicológico en los involucrados. Actualmente el $49 \%$ de las adolescentes embarazadas abandonan la escuela a lo largo del ciclo. escolar y es en este periodo cuando la familia tiene un papel importante al ser el primer escalón de apoyo de la red social, ya que es en ella donde se definen las oportunidades de crecimiento y desarrollo para el binomio.

Propósito: Explorar la experiencia de las adolescentes sobre el apoyo familiar durante el embarazo en una escuela de nivel medio superior, como parte de un fenómeno negociado para la continuación de los estudios.

El diseño fué una triangulación metodológica, en donde cuantitativamente se estableció un estudio descriptivo, explicativo y transversal. El universo correspondió a 1,022 alumnos de una escuela de nivel medio superior de la Cd. de México, con una muestra por conveniencia de 30 adolescentes entre 15 y 19 años, que cursaran el segundo o tercer trimestre de embarazo, con inscripción regular y que vivieran con su familia de origen. Cualitativamente se entrevistaron a 9 informantes.

El procedimiento consistió en aplicar el "APGAR familiar" (Smilkenstein, 1978), la "Cédula de identificación" y la "Ficha de composición familiar", mismos que se analizaron a través de cuadros y gráficas. Paralelamente se realizaron las entrevistas a los informantes hasta lograr la saturación del concepto, mismas que fueron gravadas, transcritas, codificadas y analizadas en una matriz diseñada por las autoras para tal fin. Se procedió a la codificación axial, tras el análisis de texto, hasta obtener 8 categorías.

Resultados/hallazgos. Las adolescentes en poco menos del 50\% de los casos se concentró en los 18 años de edad. El 75\% refirió algún grado de disfunción familiar. El apoyo familiar reportó 8 categorias: 1) tipo de apoyo y personaje que lo proporciona: en donde existen las dimensiones moral (ofrecido por ambos padres), de orientación y cuidado (por la madre), económico (padre) y de complicidad y compañia (hermanos y padre de su hijo), 2) Aspectos que comunica la familia a la adolescente (expectativas del bebé), 3) figura familiar con la que mantiene comunicación (madre), 4) sentimientos que genera el embarazo en la familia ante la notificación, 5) repercusiones del embarazo en la adolescente (actividades lúdicas, en la vida diaria, domésticas, emocionales), 6) sensaciones de la joven durante el embarazo, 7) repercusiones del embarazo en la familia (mejoría en las relaciones familiares y sin cambios) y 8) reacción del padre del hijo de la adolescente ante el embarazo (sugerencia de aborto, alejamiento-abandono y posibilidad de matrimonio.

Conclusiones y discusión. Es indudable que la familia es el escenario donde se desarrolla el embarazo, sin embargo 7 de cada 10 tienen algún grado de disfunción lo que debe ser tomado en cuenta para el cuidado a la salud de las adolescentes. Contrariamente a lo que la literatura señala, las futuras madres no reportaron crisis familiar, sin embargo no se pierde de vista que los sujetos de investigación ya habian logrado negociar con la familia el continuar en ella y estudiando. La experiencia de apoyo familiar en la mentalidad de la joven atiende a modalidades muy específicas y asignadas a ciertos personajes, que de no existir sin duda dejan en entre dicho el recurso para afrontar los cambios del mismo. Por otro lado la experiencia del embarazo moviliza en sensaciones y roles tanto a la joven como a los miembros de la familia, marco sobre el cual se deben diseñar estrategias para el cuidado.

PALABRAS CLAVES: Embarazo, a adolescencia, apoyo familiar, funcionalidad familiar. 


\section{Abstract}

Latin America observes a rate of approximated annual fecundity of 100 childbirths by thousand women, between the ages of 15 and 19. In Mexico every year 450 thousand pregnancies of adolescent mothers occur, which represents $25 \%$ of the total gestations that are registered in all the country, and it carries a high social, biological and psychological impact in the involved people. Nowadays, $49 \%$ of the pregnant adolescents leave the school throughout the period and is in this stage where the family has an important role, being the primary support in the social network, since it is where the binomial opportunities of growth- development is defined.

The purpose of this study is to explore the experience of the adolescents on the family support during pregnancy in a medium-high level school, as part of a negotiated phenomenon for the continuation of the studies.

The design was a methodological triangulation, where quantitatively a descriptive, explanatory and cross-sectional study wos settled down. The universe corresponded to 1,022 students of a medium-high level school in Mexico City, the study subjects were 30 adolescents between 15 and 19 years, in the second or third trimester of pregnancy, with regular inscription and living with their origin family. Qualitatively 9 informants were interviewed.

The procedure consisted in applying the "family APGAR" (Smilkenstein, 1978), "Certificate of identification" and "Card of familiar composition", that were analyzed through graphs and tables. At the same time, the interviews to the informants were carried on until obtaining the saturation of the concept, and then taxed, transcribed, codified and analyzed in a matrix designed by the authors for such goal. The next step was the axial codification, after the text analysis, until obtaining 8 categories.

Results. The adolescents in little less than $50 \%$ of the cases were 18 years old. The $75 \%$ referred some degree of familiar dysfunction. The familiar support reported 8 categories: 1) Type of support and character that provides it: in where there are several aspects: moral (offered by both parents), of orientation and taken care of (by the mother), economic (father) and of complicity and company (brothers and sisters and father of her son), 2) Aspects that the family communicates to the adalescent (baby expectations), 3) family member with which she maintains communication (mother), 5) consequences of the pregnancy in the odolescent (free-time, in the daily life, domestic, emotional activities), 6) feelings of the young mother during the pregnancy, 7) consequences of the pregnancy in the family (improvement in the familiar relations and no changes), and 8) Reaction of the adolescent son's father before the pregnancy (proposal of abortion, distance-abandonment and possibility of marriage.

Conclusions and discussion. It is undeniable that the family is the setting where the pregnancy is developed, nevertheless 7 out of 10 have some degree of dysfunction which must be taken into account for the health care of the adolescents. Contrary to what references indicate, the future mothers did not report family crisis, nevertheless is not overlooked that the investigation subjects already had managed to negotiate with the family to continue studying and staying in the family. The experience on family support in the mind of the young mother is focused in very specific modalities and assigned to certain characters, and undoubtedly if they did not exist they would compromise this resource of confronting the changes. On the other hand the experience of the pregnancy mobilizes in sensations and roles, of the young mother and the family members, which should be token into account when designing strategies for the health care.

Key Words: Adolescent, pregnancy, family support, family functionality. 


\section{INTRODUCCIÓN}

América Latina observa una tasa de fecundidad anual aproximada de 100 partos por mil mujeres, entre 15 y 19 años.' En México cada año se dan 450, 000 embarazos de madres adolescentes lo que representa el 25\% del total de gestaciones que se registran en todo el pais, ${ }_{1}^{2}$ con un alto impacto social, biológico y psicológico en los involucrados. ${ }^{3.45}$. Actualmente el 49\% de las adolescentes embarazadas abandonan la escuela a lo largo del ciclo escolar y es en este período cuando la familia tiene un papel importante al ser el primer escalón de apoyo de la red social, ya que es en ella donde se definen las oportunidades de crecimiento y desarrollo para el binomio?. Investigaciones previas han explorado el fenómeno a través de diseños cuantitativos y desde una perspectiva epidemiológica, siendo los menos los cualitativos con rescate de la experiencia humana de estas jóvenes madres.

En este marco se planteó la siguiente pregunta de investigación: ¿cuál es la experiencia de las adolescentes sobre el apoyo familiar durante el embarazo?, con el propósito de analizar la experiencia de las adolescentes embarazadas sobre el apoyo familiar.

\section{METODOLOGÍA}

La investigación fue sustentada en la triangulación metodológica. En su parte cuantitativa el diseño fue de tipo descriptivo, explicativo y transversal. El universo correspondió 1,022 estudiantes de una escuela de nivel medio superior de la ciudad de México. La muestra fue por conveniencia, no probabilistica y estuvo constituida por 30 adolescentes entre los 15 y 19 años, estudiantes regulares, primigestas, entre el $2^{\circ}$ y $3^{*}$ trimestre, que vivieran con su familia de origen y no con el padre de su hijo. La recolección de información se basó en la fórmula CUANTITATIVO + cualitativo; en donde la submuestra cualitativa se desprendió de la muestra cuantitativa con una entrevista semiestructurada. El procedimiento consistió en aplicar tres instrumentos: el "APGAR Familiar Modificado" (Smilkstein, 1978), constituido por siete preguntas con respuestas tipo Likert, la "Ficha de Estructura Familiar" (Castro, D. L., 2004). Para el análisis de resultados se recurrió a cuadros y gráficas con frecuencias y porcentajes en Excel. Para el momento cualitativo se realizó una entrevista semiestructurada con siete preguntas eje, mismas que se grabaron previa autorización de los participantes, y se transcribieron en su totalidad.

Los informantes fueron 9 hasta llegar a la saturación del tema. Para el análisis se diseñó una matriz de análisis que permitió la codificación abierta y axial para la construcción de subcategorías y categorias.

\section{RESULTADOS}

Los resultados se presentaron en tres apartados: sociodemográficos, APGAR familiar y hallazgos cualitativos. En los sociodemográficos se reconoció que el $46.67 \%$ de las jóvenes tenían 18 años, seguidas por las de 17 $(30.00 \%)$. Los integrantes por familia osciló entre 5 y 6 miembros (30\% y $23.33 \%$ respectivamente), y sólo una familia se encontró con nueve integrantes (33.33\%).

En cuanto APGAR familiar, el 57\% de las adolescentes refirió encontrarse con disfunción leve, mientras que el 7\% señaló con disfunción severa (ver gráfica 1).

Cualitativamente se identificaron 8 categorias y 16 subcategorías en torno al apoyo familiar (ver cuadro 1).

\section{Cuadro 1}

\section{Categoría y subcategorías cualitativas}

\begin{tabular}{|c|c|}
\hline CATEGORIASS & SUBCATEGORIAS \\
\hline $\begin{array}{l}\text { 1. Tipos de apoyo } \\
\text { "percibido" por la } \\
\text { adolescente y } \\
\text { miembro de la familia } \\
\text { que lo proporciona }\end{array}$ & $\begin{array}{l}\text { - Moral } \\
\text { - Erientación/ Cuidado } \\
\text { - Comómico } \\
\text { Complicidad/ } \\
\text { Compaña }\end{array}$ \\
\hline $\begin{array}{l}\text { 2. Aspectos que } \\
\text { comunica la familia a } \\
\text { la adolescente durante } \\
\text { el embarazo }\end{array}$ & $\begin{array}{l}\text { - Expectativas sobre el } \\
\text { bebé }\end{array}$ \\
\hline $\begin{array}{l}\text { 3. Figura familiar con la } \\
\text { cual mantiene la } \\
\text { adolescente } \\
\text { comunicación durante } \\
\text { el embarazo }\end{array}$ & $\begin{array}{l}\text { - Madre } \\
\text { - Hermana } \\
\text { - Padre }\end{array}$ \\
\hline $\begin{array}{l}\text { 4. Sentimientos que } \\
\text { genera el embarazo } \\
\text { en la familia }\end{array}$ & $\begin{array}{l}\text { - Sentimientos de la } \\
\text { familia ante la } \\
\text { notificación }\end{array}$ \\
\hline $\begin{array}{l}\text { 5. Repercusiones del } \\
\text { embarazo en la } \\
\text { adolescente }\end{array}$ & $\begin{array}{l}\text { - Actividades de la vida } \\
\text { diaria } \\
\text { - Actividades lúdicas } \\
\text { - Actividades } \\
\text { - Camésticas } \\
\text { - Sin cambios emocionales en las } \\
\text { actividades }\end{array}$ \\
\hline $\begin{array}{l}\text { 6. Sensaciones de la } \\
\text { adolescente ante el } \\
\text { embarazo }\end{array}$ & \\
\hline $\begin{array}{l}\text { 7. Repercusiones del } \\
\text { embarazo en la familia } \\
\text { de la adolescente }\end{array}$ & $\begin{array}{l}\text { - Mejoría en las } \\
\text { - } \text { relaciones familiares } \\
\text { relacionbios en las } \\
\text { remiliares }\end{array}$ \\
\hline $\begin{array}{l}\text { 8. Reacción del padre } \\
\text { del hijo de la } \\
\text { adolescente ante el } \\
\text { embarazo }\end{array}$ & $\begin{array}{l}\text { - Sugerencia de aborto } \\
\text { - Alejamiento/ } \\
\text { abandono } \\
\text { - Posibilidad de } \\
\text { matrimonio }\end{array}$ \\
\hline
\end{tabular}


La categoría Tipos de apoyo percibido por la adolescente y miembro de la familia que lo proporciona, se identifica. ron 4 tipos:

a) Apoyo moral centrado en la figura de ambos padres y en donde los testimonios de algunas de ellas señalan:

"...sentimentalmente, moralmente que...que me sigan brindando el apoyo que me estaban brindando mucho antes, antes de que yo estuviera embarazada eso para mi significa mucho para mi de parte de ellos".

b) Orientación/ cuidado es desempeñado particularmente por la madre o la figura femenina o masculina que funja como tal.

"En ese, ese aspecto [el cuidado], mi mamá es la que me asesora más, ella es la que me dice sabes que no comas cosas chatarra, no comas esto porque al bebé le hace daño, no te duermas porque se va a pegar el bebé, este...no subas muchas escaleras, no te agaches, no hagas muchos corajes, porque el bebé sale enojón, ella es más que nada la que me dice más que nada toma estas precauciones, ahorita que estás embarazada debes de cuidarte más y más por el bebé, ella es la que más me, me asesora en esas cosas".

\section{c) El económico}

Con respecto a la dimensión económica es una función centrada principalmente en los varones de la familia, en donde la primera figura es el padre de la joven quien solventa los gastos de la gestación y del futuro bebé, algunas veces es el "novio" quien aporta pequeñas cantidades de dinero particularmente para cubrir gastos en torno al embarazo y en otros casos son los hermanos mayores los que contribuyen con regalos especificamente para el bebé:

"De hecho mi papá siempre ha sido él que contribuye a los gastos de la casa, incluyéndome a mi ahora que estoy embarazada, es él que contribuye a los gastos".

"Este... mi novio me apoya igualmente económicamente"

"Mis hermanos, este...bueno uno está casado, y este...dos estudian pero..si me apoyan porque, le compran cosas al bebé". d) En cuanto a la dimensión de complicidad y compañía es un tipo de apoyo que las adolescentes centran en los hermanos de edades muy próximas, y en ellos se dan los procesos de acompañamiento durante la gestación. Por otro lado, sólo hubo un caso en donde la hermana menor mostró resentimiento, lo que coincidió con una distancia mayor en edad entre ellas. Asi el discurso:

"Mi hermana, con mucho resentimiento porque era la más chica ella y yo era la mayor...y yo era su adoración, y para todos lados conmigo y entonces lo tomó muy mal ella, pero de ahi en fuera todo está bien"

En lo que respecta a la categoría: aspectos que se comunican de la familia a la adolescente; las expectativas entorno al bebé de la joven embarazada fué el principal punto; así se expreso una joven:

"..se habla mucho [del bebé] porque casi la mayoría del tiempo me andan preguntando y este...¿itu qué quieres que sea? y itu cómo quieres que se llame? y ya ipensaste en el nombre? y este... ¿cuándo lo vas a bautizar? o sea todavía no nace y ya quieren que lo bautice (ríe) pero...y si, si siempre me andan hablando de él y me dicen ¿cómo está? y si ise mueve? y todo, entonces este...si se platica mucho en mi casa de esto"

La categoría tres que aduce a figura familiar con la cual mantiene la adolescente comunicación durante el embarazo; sin lugar a dudas la femenina es con la que mas interactúan probablemente por una condición de género; los argumentos señalaron:

"No se como que siento más confianza con ella [con la mamá] porque somos mujeres, a mi mamá y mi hermana un poco"

La categoría cuatro: Sentimientos de la familia ante el embarazo, permitió reconocer que posterior a la notificación del mismo emergieron el enojo del padre, "molestias" y frustración. Aquí un testimonio:

..." Imamá] que si lo quería tener que ella me iba apoyar en lo que sea ...me dijo que si lo tuviera que la decisión era muy mía"...[papá](silencio prolongado, contenienda las lágrimas) "me dejó de hablar" (se le quiebra la voz)...."Pues...pues que era normal que un día iba a suceder algo asi en mi, si no era, ahorita era más tarde de pronto lo aceptó (baja el tono de voz).". 
Con respecto a la categoría cinco: Sensaciones de las adolescentes ante su embarazo, las jóvenes mencionaron en el discurso el cansancio físico, sentimientos de ternura hacia el futuro bebé, emoción y satisfacción:

"Me siento emocionada, se mueve mucho, saber que alguien que esté dentro de mi, que ahora él va depender de mi a ella va a depender de mi, pues me dan ganas de seguir luchando y de seguir adelante, es algo muy, muy bonito algo que no encontraría todas las palabras para especificar lo bonito que sé siente tener alguien dentro, dentro de ti".

En cuanto las repercusiones del embarazo en la adolescente se evidenciaron cinco subcategorías:

a) Actividades de la vida diaria; en este sentido refirieron una modificación importante de la cotidianeidad y lo explicaron con discursos como el siguiente:

"Si todo ha cambiado porque este...ya no, no he salido, bueno en todo mi embarazo no, no he ido a fiestas, ni a ningún lado pero... este...no me hace sentir mal porque yo se que lo hago".

Las actividades lúdicas, presentaron una disminución en cuanto a frecuencia y modificación; es decir pasaron de actividades de movimientos fuertes y amplios a una actividad reducida o minima:

"Es que ya no juego, ya no corro, ya no hago 10 mismo porque antes...ahorita pues ya no puedo hacerlo por como me evito de mi embarazo...pues han cambiando muchas cosas, ya no puedo seguir haciendo el mismo ejercicio ni las mismas actividades".

c) Las actividades doméșticas, existió un decremento en su realización.

"Antes lavaba asi, y luego mi mamá se iba a trabajar y yo este...yo y yo me quedabo a lavar y hacer el quehacer con mi hermana y ahora ya no".

d) Cambios emocionales algunas jóvenes manifestaron presentar ambivalencia entre tristeza y alegria, inclinándose más a la tristeza, aunque hubo algunas que refirieron que esto se modificó cuando "pensaron en la responsabilidad de ser madre: "...pues, si, si ha cambiado, porque, se podria decir que yo antes era una muchacha que no pensaba las cosas, que las hacía asi como ial hay se va! como cualquier otro chavo o chava joven ino?, pero ya cuando estas embarazada, o tienes un hijo te das cuenta de que las cosas no son totalmente fácil, te das cuenta que hay responsabilidades y piensas y haces las cosas con más responsabilidad".

La categorla repercusiones del embarazo en la familia de la adolescente desprendió la subcategoría mejoría en las relaciones familiares. Particularmente en comunicación, comprensión, atención, cuidados, unión familiar, he aquí uno de los testimonios:

"Este... mmmh... como que ...hubo más comunicación entre nosotros de mis papas y mis hermanos...por el embarazo porque yo digo que como va ser su primer nieto han de estar felices".

La categoria: Actitud del padre del hijo de la adolescente ante el embarazo reportó posturas tales como:

La sugerencia de aborto en donde al final la joven decidió enfrentar la situación y las consecuencias de no realizarlo:

\begin{abstract}
"Porque este...porque al fin de cuentas ya lo tenía planeado [el novio\}, yo estábamos buscando el lugar y todo, pero no sé, senti un sentimiento dentro de mi y dije no, pues como voy a matar una vida, mejor decidi hablar con mi mamá personalmente (se le quiebra la voz). sibien me aceptaba y sibien pus me iba a decir arréglatelas como tu puedas, pero al fin de cuentas si, si me acepto con el bebé".
\end{abstract}

Otra postura fué el alejamiento o abandono del padre de su hijo, en donde se registraron algunos casos en los que por diferentes razones se presentó:

"...Cuando le dije que estaba embarazada me, me dio la espolda, me dijo que él no estabo listo para tener hijos y que no contaba con apoyo económico, con nada y se fué y ya no supe nada de él".

Y finalmente la posición de un matrimonio prematuro, en donde era una forma de responder:

"Él ahorita si está conmigo pero este...él dice que si quiere vivir conmigo, casarse y así, pero yo no quiero." 


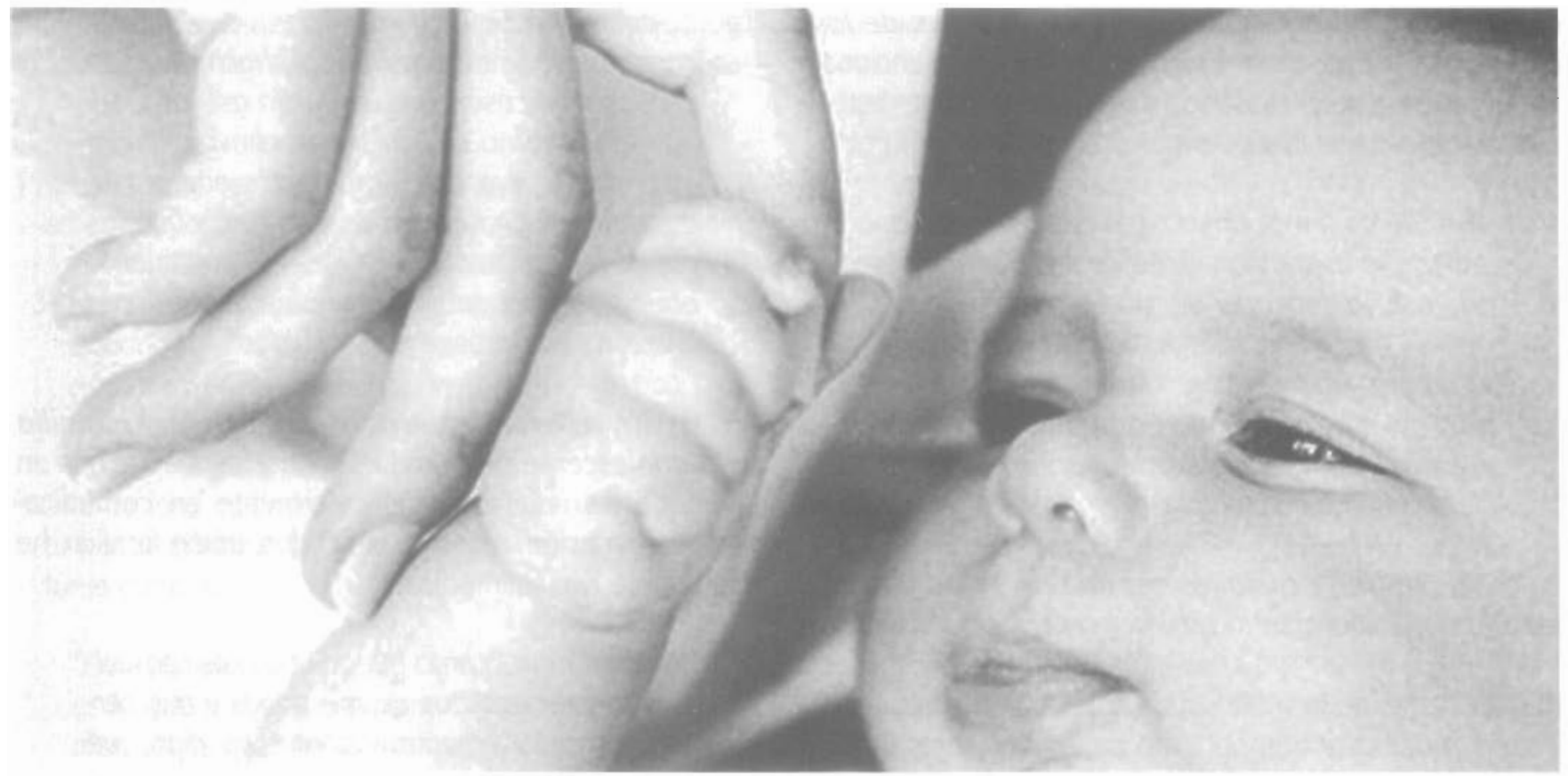

\section{CONCLUSIONES Y DISCUSIÓN}

A partir de la investigación quedó de manifiesto que la familia representa un sistema fuerte de apoyo para la joven embarazada y que existen de manera clara personajes que lo proporcionan y formas en que lo proveen.

En cuanto a la dinámica familiar, las jovenes reconocen algún grado de disfunción preexistente pero no a consecuencia de su embarazo. Sin embargo, pese al escenario familiar y después de un proceso de negociación que realiza la joven con su grupo parental, le permiten que continúe su. embarazo y sus estudios, aspecto que se explica cuando se acepta que cada miembros de la familia tiene la función de adaptarse y superar la crisis con sus propios recursos?.

En cuanto al apoyo familiar las adolescentes reconocieron cuatro dimensiones: el moral, el de orientación/cuidado, el económico y el de complicidad/compañía.

De estos, el moral es proporcionado por ambos padres, lo que concuerda con lo referido por diversos autores, 9.10 en el sentido de este apoyo moral lo brinda la familia y es visto como el grado en el que una adolescente soltera, primípara cree que sus necesidades de afecto, información y retroalimentación fueron llenadas por su autodefinida familia de origen".

El apoyo de orientación y cuidado se depositó en la madre o un personaje femenino, aspecto que se ve confirmado con lo que otros autores señalan en el sentido de el mantenimiento de la salud y el bienestar de la mujer durante el proceso reproductivo mantiene una estrecha relación con el apoyo casi siempre femenino ${ }^{12.13}$.

Ocasionalmente existe un desplazamiento de este rol hacia el padre de la joven cuando es este el que pasa más tiempo con ella. Lo que cuestiona el que realmente la orientación y cuidado sea una "funciónn" de género y hace suponer que está determinado en mucho por el contacto personal y continuo entre las partes.

En lo que respecta al apoyo económico, los padres de las jovenes siguen cumpliendo principalmente como proveedores monetarios porque las adolescentes siguen presentado una situación de dependencia, aspecto en ha sido confirmado por otros ${ }^{14}$ 15.6. Con respecto a los hermanos coetáneos son los que proporcionan el apoyo de compañía y complicidad, ratifican lo anterior ${ }^{17}$.

Llama la atención que una gran proporción de las entrevistadas mencionaron sentirse tranquilas durante el embarazo, esto posiblemente por sentir el apoyo del su grupo familiar en varias dimensiones incluso llegando a tomar conciencia de la responsabilidad que implica la maternidad. Esto corresponde con lo mencionado por Kano, 1999, de que al decidir continuar con el embarazo la joven hace tránsito a una etapa adulta al realizar un compromiso irreversible e inicia la construcción de una nueva identidad, "ser madre" 2 . 


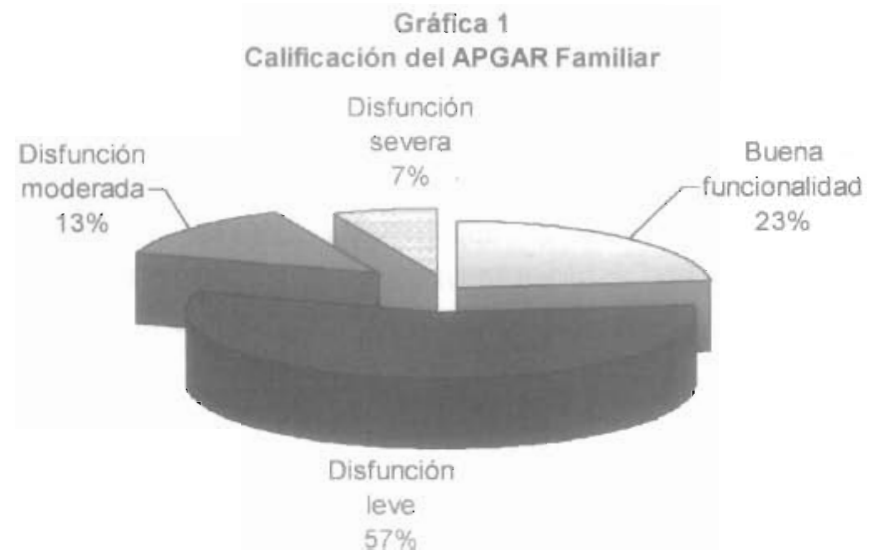

Referente a las repercusiones del embarazo en la familia de la adolescente, uno de los hallazgos más importantes de la investigación es que a cuando la joven inicia una negociación con los integrantes de su grupo parental las relaciones familiares mejoran y la "crisis familiar" al que hacen referencia diferentes autores y que afirmaron que la familia entera entra y se mantiene en crisis con el embarazo de la adolescente ${ }^{1020}$, aunque en este caso se resolvió dentro del propio seno de manera diferentes.

Sugerencias: Estas se centrarían en contemplar en nuevas aproximaciones al fenómeno de la experiencia de las jóvenes que no pudieron negociar el continuar con sus estudios y con su familia parental. Por otro lado, explorar la perspectiva del apoyo por parte de los padres durante el embarazo adolescente.

Para terminar comentaremos que la experiencia de apoyo familiar en la mentalidad de la joven atiende a modalidades muy específicas y asignadas a ciertos personajes, que de no existir sin duda dejan en entre dicho el recurso para afrontar los cambios del mismo. Por otro lado, la experiencia del embarazo moviliza en sensaciones y roles tanto a la joven como a los miembros de la familia, marco sobre el cual se deben diseñar estrategias para el cuidado.

\section{REFERENCIAS BIBLIOGRÁFICAS}

1. De la Cuesta- B; Carmen. Significado del embarazo en la adolescencia: la dimensión sentimental. En Enfermera Clinica. Vol 10 No. 5 septiembre-octubre. México, 2000, p.26-31.

2. Instituto Nacional de Estadistica, Geografla e Informática (2001). Estadisticas de salud reproductiva. Recuperado 08/12/04, de http://negi.gob.mx/est/contenidos/espanol/tematicos/mediano/me: d.asp? =mpob038\&c=3180.
3. Atkin- C; Lucille, Pick- W. Susan. Antecedentes Psicosociales del Embarazo en la Adolescencia. En Revista de Perinatologia de Reproducción Humana. Vol 3 No 3 julio-septiembre. México, 1989. p. $152-158$.

4. Espinoza- M; Alfredo, Anzures- L; Beatriz. Sección Bibliográfica: Adolescentes. En Revista Médica del Hospital General. Vol 62 No 3 julio-septiembre. México, 1999. p $210-215$

5. Fernández. P; Francisco y cols. Características sociofamiliares y consecuencias en la salud materno-infantil del embarazo precoz. En Bol Med Hosp Infant Mex. Vol 53 No 2 febrero. México, 1996. p 84 88

6. Maddaleno- M; German, Munist- M; Mabel, Serrano- V; Carlos. Lo Salud de la Adolescente y del Joven. Ed. Organización Panamericana de la Salud. México 1996. 243 pp.

7. Ortigasa- C; Eduardo, Padilla- 1. Yolanda \& López- O. Rocio. Necesidades educativas en salud perinatal en madres adolescentes embarazados. En Ginecología y Obstetricia de México. Vol 70 enero. México 2002. p.28-34

8. Homilt- C Nina; Florenzano- U. Raman. \& Ringeling- P. Isabel. Familid y salud familiar, un entoque pora la atención primaria. En Boletin de la Oficina Sanitaria Panamencana. Vol.48 No 2 febrero 1995. p 144. 154.

9. Kudakwashe, Gerard. \& Andrews- Martin. La influencia de los factores de condicionamiento bósicos de la familia y losiamigos, y de la acción de autocuidado sobre el compromiso de adolescentes solte ras primiparas con la práctica anticonceptiva. En Journal of Community Health Nursing. Vo! 12 No2: México, 1995 p.89-100.

Linares- A; Manuel. \& Gálvez- T; Alberto. La relación de ayuda a la mujer durante el embarazo, parto y puerperio del primer hijo. En Index Vol 38, No 9, España 2002.

10. Kudakwashe, Gerard. \& Andrews-Martin. opcit

12. Dulanto- G. Enrique. El adolescente. Ed McGraw-Hill Interamericana. México 2000. 538 pp.

13. Ortigasa- C; Eduardo, Padilla- J. Yolanda \& López- O. Rocio. opcit:

14. Reeder- J; Sharon, \& Martin- L. Leonide. Enfermeria Materno-intontil. Ed. Harla. 16a ed. México 1995-90pp.

15. Burroughs- Arlene. Enfermerio materno infantil. Ed, Mc Graw-Hill Interamericana 5a ed. México 1998. 8 pp

16. Masters- Willian, Jonson- Violet. \& Kolodny-. Richard. La sexuolidad' humana. Tomo 2; México 1998

17. Novel- M; Gloria, Lluch- C; Teresa \& López-V. Dolores. Enfermería psicosocial. Ed Serie Manuales de Enfermería. Barcelona, España 1991.83 pp.

18. Kano- F. Esther. El Proceso de Enfrentar un Embarazo en la Adolescencia: Ajustando la Identidad. En Investigación y Educación. en Enfermeria: Vol 13. No.1, marzo, México 1999. p. 35-46.

19. Stern-Claudio. El Embarazo en la adolescencia como un problema público: una visión crítica. En Salud Pública de Mexico, Vol. 39, No.2 marzo-abril 1997 p. 137-143.

20. Veloza, M. Martha. (). Comparación del grado de salud familiar en familias con adolescentes gestantes can presencia o no de morbilidad en el tercer trimestre del embarazo. Tesis de Maestría de Enfermería no publicada, Universidad Nacional de Colombia, Colombia 2003. 7pp

\section{DIRECCIÓN PARA CORRESPONDENCIA}

Leticia Castro: leticiacd2002@yahoo.com.mx

Rosa Ma. Ostiguin: rosyostiguin@hotmail.com. 